\title{
Subtraction ICG angiography in Harada's disease
}

Takeya Kohno, Tokuhiko Miki, Kunihiko Shiraki, Kiyoshi Kano, Michiko Matsushita, Kazuhiko Hayashi, Jean Jacques De Laey

\begin{abstract}
Backgroundlaim-The significance of indocyanine green (ICG) angiography (ICGA) in Harada's disease still awaits clarification in many respects. This study investigates the details of choroidal lesions observed in Harada's disease by the subtraction method.

Methods-Eight patients with Harada's disease were followed with ICGA. ICG angiograms were obtained with a Topcon high resolution digital fundus camera and processed with a Topcon IMAGEnet computer system. Image subtraction was conducted for analysing serial angiograms taken at about 2 second intervals during the dye transit phase and those taken in the early and middle phases of angiography.
\end{abstract}

Results-Standard ICG images of acute stage disease showed delayed choroidal filling in the early phase. Mid phase angiograms showed areas with bright fluorescence of variable intensity, indicating intrachoroidal ICG leakage. With image subtraction of angiograms with an interval of seconds the choroidal vessels could be imaged sequentially, with the choroidal arteries visualised first, followed by the definition of the choriocapillaris and then the choroidal veins. The choroidal veins with delayed filling were visualised as positive images in serial subtraction angiograms. Subtraction with an interval of minutes showed uneven background fluorescence and bright fluorescence corresponding to the areas of intrachoroidal ICG leakage. After the disease subsided with steroid therapy, angiography revealed an improvement in delayed choroidal filling. Image subtraction by the second allowed a clear visualisation of improved choroidal venous filling, while subtraction by the minute showed homogeneous background fluorescence, eliminating brighter areas.

Conclusion-Subtraction ICGA demonstrated that delayed filling of the choroidal veins of varying severity occurs in association with hyperpermeability of the choroidal vessels in the course of Harada's disease.

(Br f Ophthalmol 1999;83:822-833)

Correspondence to: Dr Takeya Kohno, Department of

Ophthalmology, Osaka City

University Medical School,

Asahi-machi, 1-4-3,

Abeno-ku, Osaka, 545-8585

Japan.

Accepted for publication 1 March 1999

The development of indocyanine green angiography (ICGA) has made it possible to examine choroidal vascular lesions in detail. ${ }^{1}$ ICGA has been reported to allow enhanced visualisation of intrachoroidal lesions, such as the choroidal vessel irregularities in Harada's disease, which are not visible with conventional ophthalmoscopy or fluorescein angiography (FA), indicating its utility in diagnosing and evaluating treatment of the disease..$^{3-8}$

However, the interpretation of ICGA findings is difficult for several reasons: (1) since the structure of the choroidal vasculature is three dimensional, fluorescence from vessels in different layers overlaps, producing complicated images; (2) the dye flows into the choroidal vessels too quickly to allow accurate assessment as to whether choroidal filling is normal or delayed; and (3) equipment for ICGA is designed basically to adjust the intensity of the emission light according to the brightness of the image obtained, often making it difficult to determine if the observed fluorescence is excessive or normal. Thus, findings from ICGA studies of Harada's disease cannot as yet be considered conclusive.

Application of the subtraction method, a computer assisted image processing technique which subtracts one frame from another, in the analysis of ICGA permits detailed examination of circulatory changes in the choriocapillaris and choroidal veins ${ }^{910}$ and objective assessment of ICG leakage..$^{10}{ }^{11}$ In the current study, we performed ICGA in patients with Harada's disease and analysed the angiographic findings using the image subtraction method.

\section{Methods}

The subjects were eight patients diagnosed as having Harada's disease and followed with ICGA for at least 6 months at the department of ophthalmology of Osaka City University Medical School from April 1993 to December 1997. The diagnosis was based on clinical symptomatology, ophthalmoscopic and FA findings, and clinical laboratory test results of HLA haplotypes, especially HLA DR4. The subjects underwent a full ocular examination, including measurement of visual acuity, slit lamp examination, and funduscopy.

FA was performed with the Topcon 50-IA fundus camera and ICGA utilised a high resolution digital infrared fundus imaging system (Topcon H1024 high resolution digital camera, Topcon Corp, Tokyo, Japan). ICG angiograms were taken serially at intervals of 2 seconds during the dye transit phase, and thereafter at 2, 5, 10, 20, and 30 minutes. The angiograms were stored on a magnetic optical disk as digital images with a resolution of 1024 $\sim 1024$ pixels (256 grades of grey scale).

Subtraction image analysis was performed with a Topcon IMAGEnet computer system using a method previously described. ${ }^{10}$ Briefly, two 

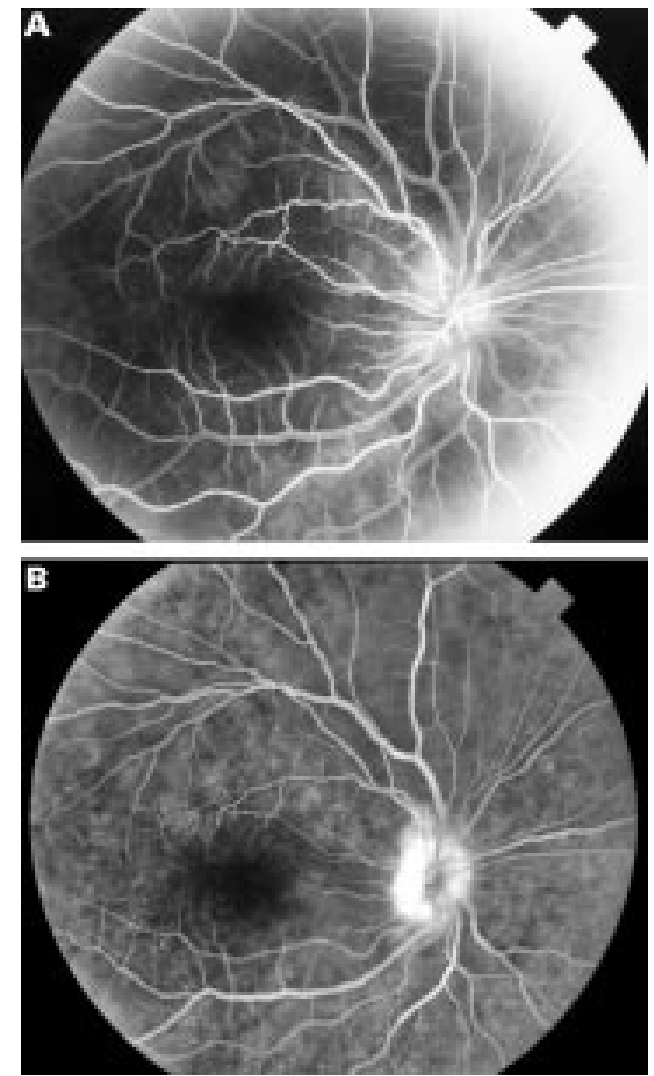

Figure 1 Case 3. Before steroid therapy. FA. (A) At 27 seconds, delayed choroidal filling is noted. (B) At 2 minutes, numerous hyperfluorescent dots are observed.

different images taken at intervals of either seconds or minutes were selected depending on the purpose. In the present study, subtraction analysis was made between the serial angiograms with an interval of seconds in the dye transit phase and between the angiograms with an interval of minutes by subtracting images taken in the early phase (about 30 seconds) from those taken in the middle phase (at 10-15 minutes). The images were aligned by marking identical points on each image, and subtraction was done between the images at each pixel. In the subtracted images, the area without difference in brightness between the two images was visualised as medium grey using 128 grades. Elimination of grey scales darker than the 128th grade provided an image that showed changes during the interval of imaging. Thus, the positive images were defined as those brighter on later frames than earlier frames while negative images were brighter in the earlier than in the later frames. Subsequently, contrast was adjusted to meet the purpose of the analysis.

\section{Results}

The eight cases examined in this study are summarised in Table 1. There were five men and three women aged between 20 and 64 years (mean 42.0 years). All patients reported visual impairment in the acute stage of the disease, but the subjective symptoms subsided after steroid therapy in most cases. Intraocular pressure was within normal range in every case.
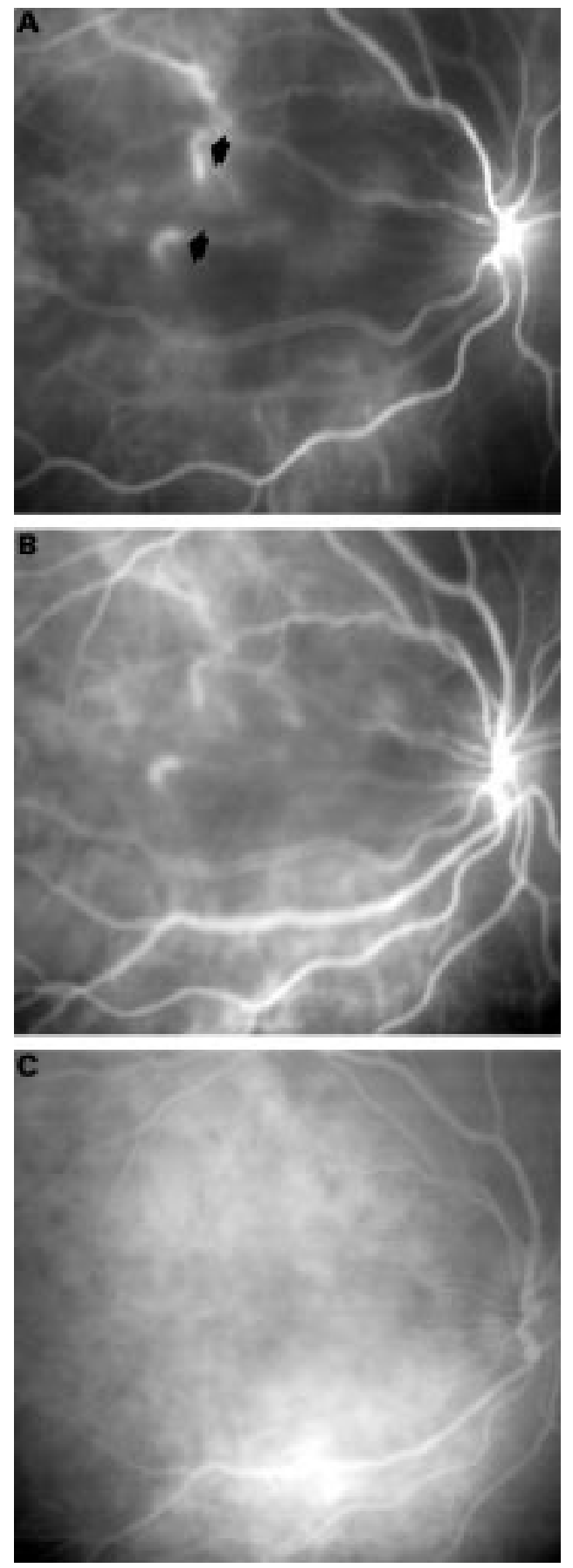

Figure 2 Case 3. Before steroid therapy. Standard ICGA. (A) 26.9 seconds, (B) 35.2 seconds. Choroidal arteries are visualised, followed by visualisation of the choriocapillaris and choroidal veins. Visible choroidal arteries are dilated and tortuous (arrows). The course of the choroidal vessels is irregular and the number of visible vessels decreased. $(C)$ 14 minutes 49.4 seconds. The contour of the choroidal vessels is obscured. Hyperfluorescence of varied brightness is seen over the entire posterior pole, including areas without the punctate hyperfluorescent lesions noted on FA.

In the acute stage, numerous punctate hyperfluorescent lesions caused by dye leakage were noted on FA in six of eight cases. In the other two cases, only leakage on the optic disc was observed. In both the acute and recovery stages, early and mid ICGA findings were similar in all patients. The typical findings on FA and ICGA were demonstrated in representative cases described below. 

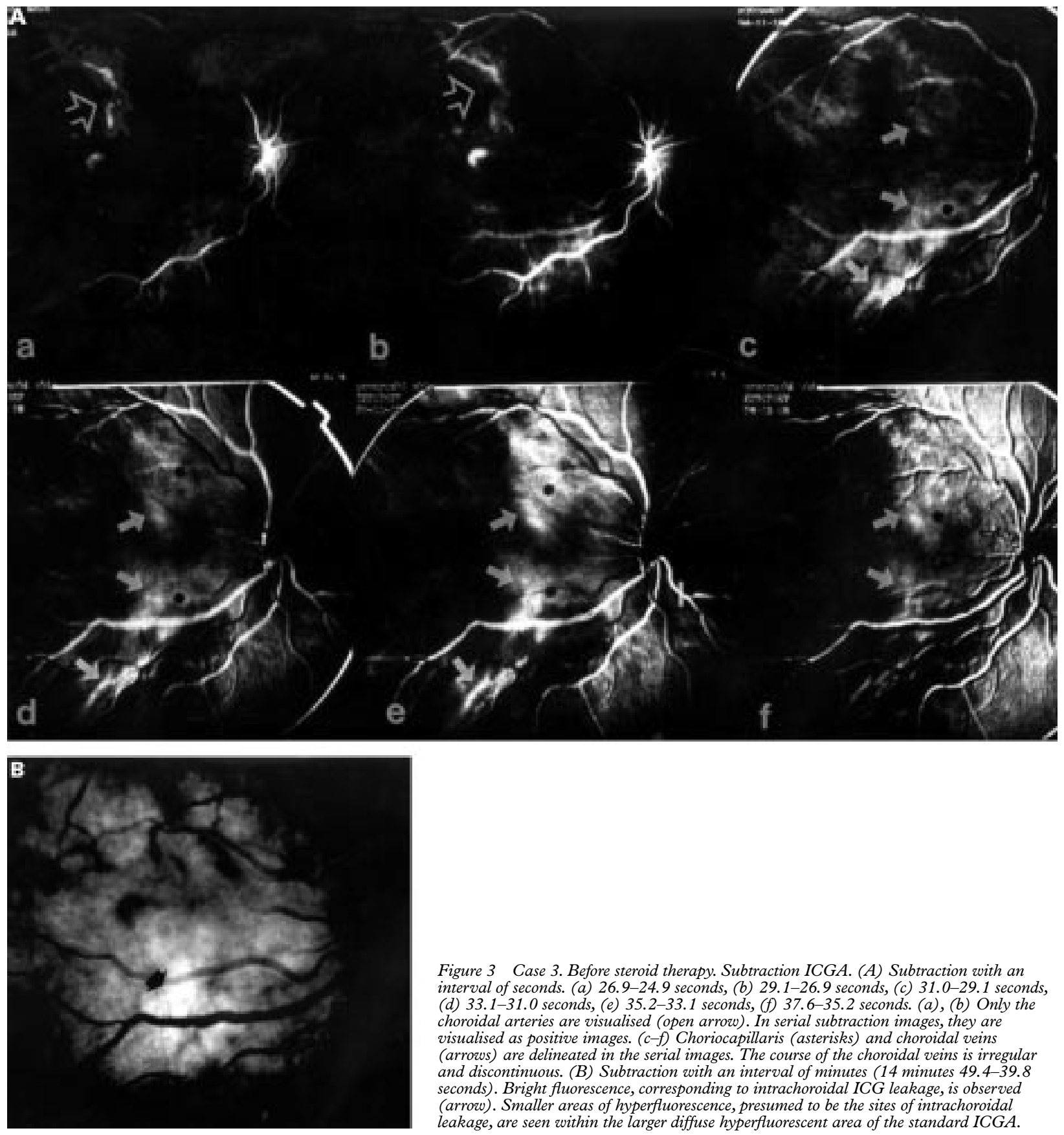

Figure 3 Case 3. Before steroid therapy. Subtraction ICGA. (A) Subtraction with an interval of seconds. (a) 26.9-24.9 seconds, (b) 29.1-26.9 seconds, (c) 31.0-29.1 seconds, (d) 33.1-31.0 seconds, (e) 35.2-33.1 seconds, (f) 37.6-35.2 seconds. (a), (b) Only the choroidal arteries are visualised (open arrow). In serial subtraction images, they are visualised as positive images. (c-f) Choriocapillaris (asterisks) and choroidal veins (arrows) are delineated in the serial images. The course of the choroidal veins is irregular and discontinuous. (B) Subtraction with an interval of minutes (14 minutes 49.4-39.8 seconds). Bright fluorescence, corresponding to intrachoroidal ICG leakage, is observed (arrow). Smaller areas of hyperfluorescence, presumed to be the sites of intrachoroidal leakage, are seen within the larger diffuse hyperfluorescent area of the standard ICGA.

Table 1 Summary of cases

\begin{tabular}{lllllll}
\hline Case No & Age & Sex & $\begin{array}{l}\text { Interval between initial } \\
\text { symptom and initial } \\
\text { angiography }\end{array}$ & $\begin{array}{l}\text { Corrected visual acuity on } \\
\text { initial angiography }\end{array}$ & $\begin{array}{l}\text { Follow up } \\
\text { (months) }\end{array}$ & $\begin{array}{l}\text { Corrected visual acuity } \\
\text { on final follow up } \\
\text { examination }\end{array}$ \\
\hline 1 & 64 & F & 10 days & RV 0.1, LV 0.2 & 45 & RV 0.5, LV 0.6 \\
2 & 29 & M & 14 days & RV 0.3, LV 1.0 & 36 & RV 1.0, LV 1.2 \\
3 & 56 & M & 24 days & RV 0.9, LV 0.7 & 25 & RV 1.5, LV 1.2 \\
4 & 29 & F & 9 days & RV 0.3, LV 0.1 & 25 & RV 1.2, LV 1.0 \\
5 & 38 & F & 3 days & RV 30 cm/nd, LV 0.9 & 25 & RV 1.2, LV 1.2 \\
6 & 58 & M & 10 days & RV 1.0, LV 1.0 & 24 & RV 1.5, LV 1.5 \\
7 & 20 & M & 21 days & RV 0.5, LV 0.5 & 12 & RV 1.0, LV 1.0 \\
8 & 42 & M & 1 month & RV 0.5, LV 0.5 & 8 & RV 1.0, LV 1.0 \\
\hline
\end{tabular}


Case 3

A 56 year old man presented with headache, tinnitus, and bilateral blurred vision. Corrected visual acuity was 0.9 in the right eye and 0.7 in the left eye. Intraocular pressure was right eye $14 \mathrm{~mm} \mathrm{Hg}$ and left eye $15 \mathrm{~mm} \mathrm{Hg}$. Slit lamp examination revealed cells in the anterior chamber. Serous retinal detachment was observed in the posterior pole of both eyes; both optic discs were reddish and swollen. Ultrasonography indicated thickening of the choroid. FA revealed delayed choroidal filling in the early phase and numerous punctate hyperfluorescent lesions due to dye leakage in the mid phase (Fig 1). On standard ICGA, the retinal arteries were visualised first, followed by the choroidal arteries. The visible choroidal arteries were dilated and tortuous (arrows). The choroidal vessels, showing irregular courses, were decreased in number. In the angiogram at 5 minutes, the contour of the choroidal vessels was obscured and hyperfluorescent areas of variable brightness were seen over the whole region of the posterior pole, including the areas without the punctate hyperfluorescent lesions on FA (Fig 2). By subtraction of angiograms in the dye transit phase with an interval of seconds, the choroidal vessels could be imaged sequentially, with choroidal arteries visualised first, followed by definition of choriocapillaris and then choroidal veins, suggesting a delay of choroidal arterial filling. Choroidal veins also showed delayed filling and were visualised as positive images in serial subtraction images (Fig 3A). Subtraction with an interval of minutes revealed hyperfluorescent areas corresponding to intrachoroidal ICG leakage (Fig 3B). After 30 days on steroid therapy, the serous retinal detachment was no longer observed. The standard ICG angiogram showed an increased number of visible choroidal vessels and an improvement in the irregularity and dilatation of the choroidal vessels. The heterogeneous hyperfluorescence seen in the posterior pole in the middle and late phases had disappeared (Fig 4). On subtraction of ICG angiograms with an interval of seconds, the choroidal arteries were visualised in the initial image in the dye transit phase and were noted to be less irregular and increased in number. The delayed choroidal venous filling observed in the acute stage of disease remained (Fig 5A). On subtraction by the minute, a relatively homogeneous fluorescence was seen except in the region along the course of vessels; bright fluorescence suggestive of intrachoroidal ICG leakage was not observed (Fig 5B). After 4 months, choroidal filling had improved on FA. On standard ICGA, the retinal and choroidal arteries (arrows) were visualised simultaneously. The number of choroidal vessels had increased. The course of the choroidal veins straightened and became continuous from the posterior pole to the peripheral region, with less variation in caliber (Fig $6 \mathrm{~A})$.

On subtraction ICGA by the second, the choroidal veins were delineated in the initial picture. In addition, serial pictures clearly
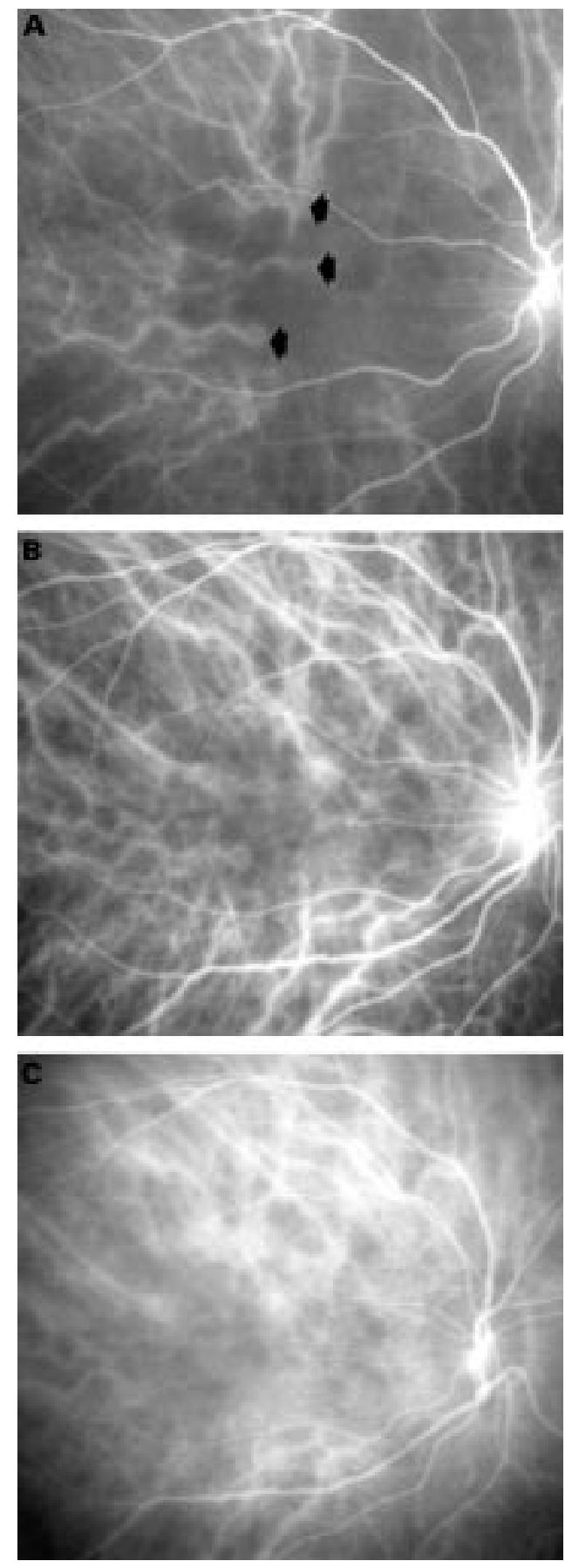

Figure 4 Case 3. One month on steroid therapy. Standard ICGA. (A) 29.9 seconds, (B) 42.7 seconds. Retinal and choroidal arteries are visualised simultaneously. The number of choroidal vessels has increased; the irregularity and calibre of the choroidal arteries have improved (arrows). (C) At 13 minutes 46.8 seconds, the contour of the choroidal vessels has become more clear and the bright fluorescence has disappeared.

demonstrated an improvement in choroidal venous filling (Fig 6B).

\section{Case 7}

A 20 year old man presented with headache, tinnitus, and bilateral blurred vision. Corrected visual acuity was 0.5 in both eyes. Intraocular pressure was right eye $14 \mathrm{~mm} \mathrm{Hg}$ and left eye $14 \mathrm{~mm} \mathrm{Hg}$. Slit lamp examination revealed cells in the anterior chamber. Serous retinal 

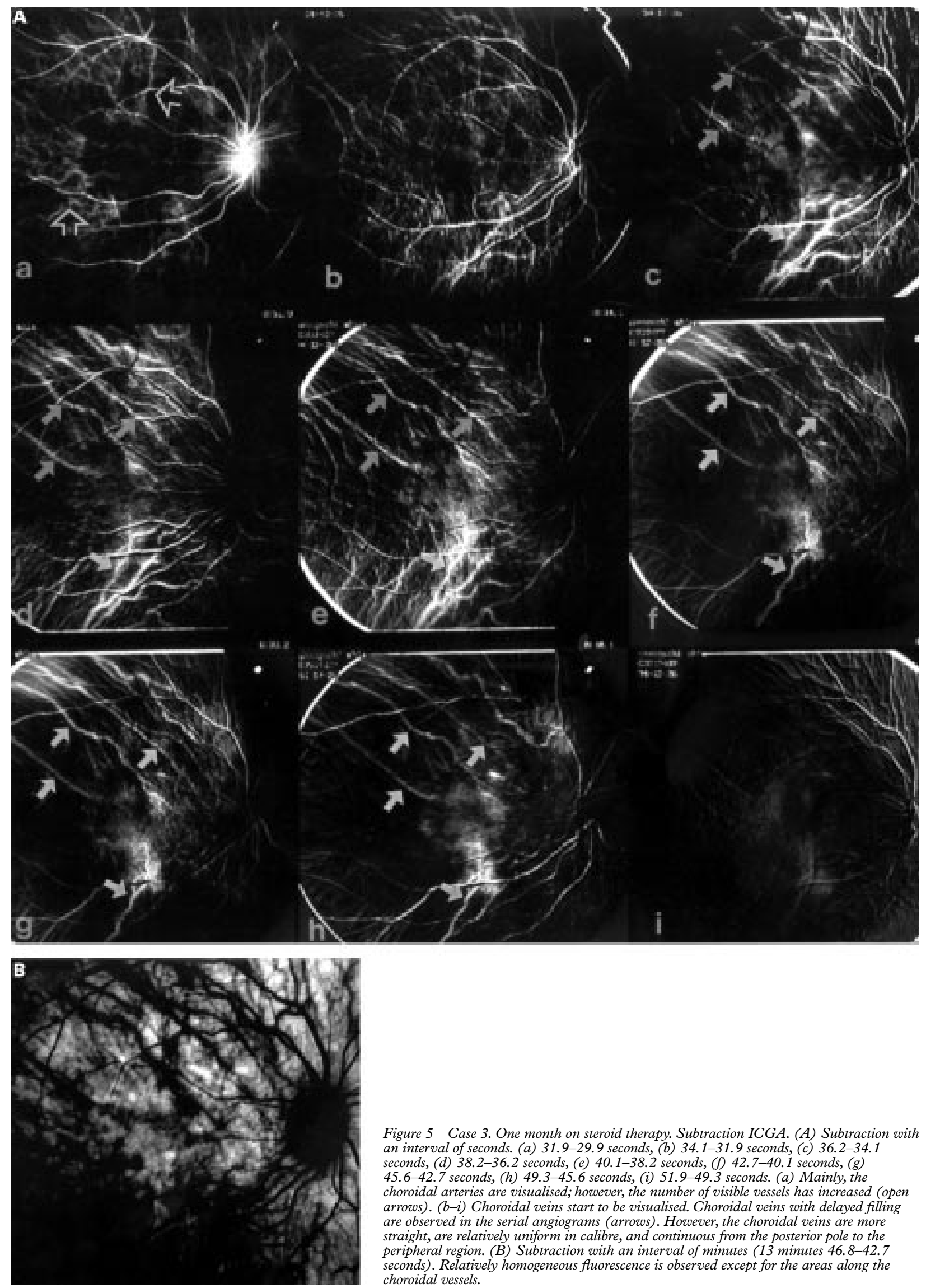

Figure 5 Case 3. One month on steroid therapy. Subtraction ICGA. (A) Subtraction with an interval of seconds. (a) 31.9-29.9 seconds, (b) 34.1-31.9 seconds, (c) 36.2-34.1 seconds, (d) 38.2-36.2 seconds, (e) 40.1-38.2 seconds, (f) 42.7-40.1 seconds, (g) 45.6-42.7 seconds, (h) 49.3-45.6 seconds, (i) 51.9-49.3 seconds. (a) Mainly, the choroidal arteries are visualised; however, the number of visible vessels has increased (open arrows). (b-i) Choroidal veins start to be visualised. Choroidal veins with delayed filling are observed in the serial angiograms (arrows). However, the choroidal veins are more straight, are relatively uniform in calibre, and continuous from the posterior pole to the peripheral region. (B) Subtraction with an interval of minutes (13 minutes 46.8-42.7 seconds). Relatively homogeneous fluorescence is observed except for the areas along the choroidal vessels. 

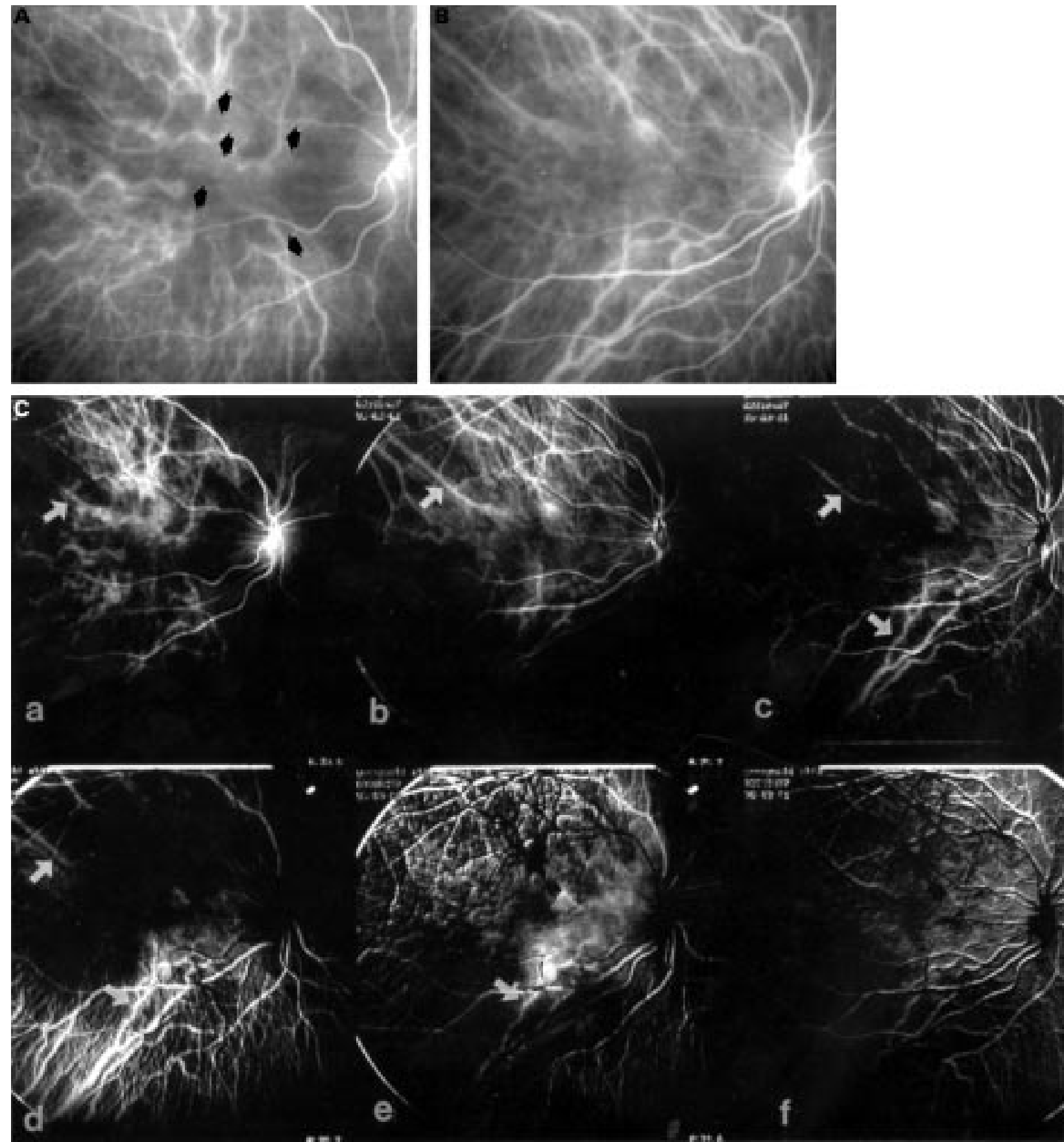

Figure 6 Case 3. Four months on steroid therapy. (A, B) Standard ICGA. (A) 21.8 seconds, (B) 48.1 seconds. Retinal and choroidal arteries were visualised simultaneously. The number of visible choroidal arteries (arrows) have increased in the initial image. The course of the choroidal veins has straightened and has become continuous from the posterior pole to the peripheral region, with less variation in calibre. (C) Subtraction ICGA. (a) 23.8-21.8 seconds, (b) 25.7-23.8 seconds, (c) 27.7-25.7 seconds, (d) 29.7-27.7 seconds, (e) 31.6-29.7 seconds, (f) 33.7-31.6 seconds. Choroidal veins (arrow) as well as the arteries are visualised in the initial subtracted image. Choroidal veins with delayed filling are observed in the serial angiograms (arrows). However, the degree of filling has improved.

detachment was not seen in the posterior pole. Both optic discs were reddish and swollen. Ultrasonography indicated thickening of the choroid. FA revealed delayed choroidal filling in the early phase and punctate hyperfluorescent lesions due to dye leakage in the mid phase (Fig 7). On standard ICGA, the retinal arteries were visualised first, followed by the choroidal arteries. Choroidal filling delay corresponding to the watershed zone was clearly observed due to hypoperfusion in the choroid. The choroidal vessels, showing irregular courses, were decreased in number. In the angiogram at 10 minutes, ICG leakage was clearly seen temporal to the optic disc beyond the area of punctate hyperfluorescent lesions on FA (Fig 8). By subtraction of angiograms in the dye transit phase with an interval of seconds the choroidal vessels could be imaged sequentially, with choroidal arteries visualised first, followed by definition of choriocapillaris and then choroidal veins. Choroidal veins showed delayed filling and were visualised as positive images in serial subtraction images (Fig 9A). Subtraction with an interval of minutes revealed hyperfluorescent areas 

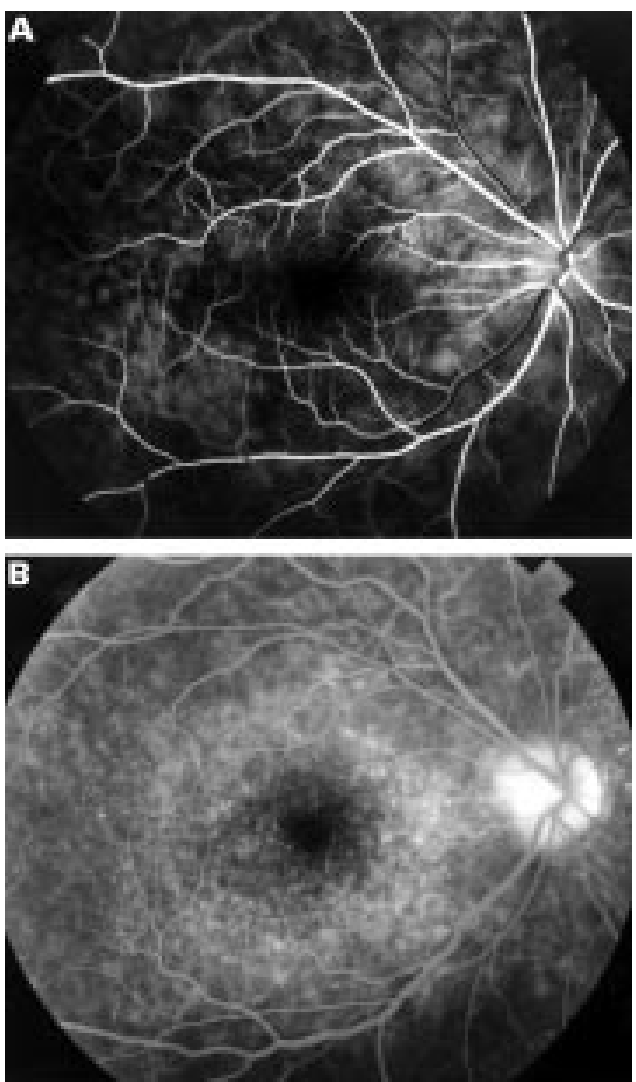

Figure 7 Case 7. Before steroid therapy. FA. (A) At 15 seconds, delayed choroidal filling is noted. (B) At 6 minutes, numerous hyperfluorescent dots are observed.

corresponding to intrachoroidal ICG leakage (Fig 9B). After 10 months of steroid therapy, the standard ICGA showed an increased number of visible choroidal vessels and an improvement in the irregularity of the choroidal vessels. The watershed zone was no longer observed (Fig 10A). On subtraction ICGA by seconds, the choroidal veins were visualised in the initial image and were noted to be less irregular and increased in number. The delayed choroidal venous filling observed in the acute stage of disease had improved (Fig 10B).

Case 8

A 42 year old man was seen with bilateral blurred vision. The corrected visual acuity was 0.5 in both eyes. The intraocular pressure was right eye $18 \mathrm{~mm} \mathrm{Hg}$ and left eye $18 \mathrm{~mm} \mathrm{Hg}$. The anterior chamber was shallow in both eyes. No cells were found either in the anterior chamber or in the vitreous of either eye by slit lamp examination. The optic discs were markedly reddish and swollen; serous retinal detachment, though observed in the posterior pole, was mild. Steroid therapy with betamethasone $16 \mathrm{mg}$ was administered, followed by tapering. The patient was maintained on the tapered dose of $2 \mathrm{mg}$ of betamethasone. After 4 months of steroid treatment, however, papilloedema in the right eye was exacerbated. Ultrasonography revealed marked thickening of the choroid in both eyes. On FA, although hypofluorescence giving the choroid a folded appearance was seen, hyperfluorescence from dye leakage
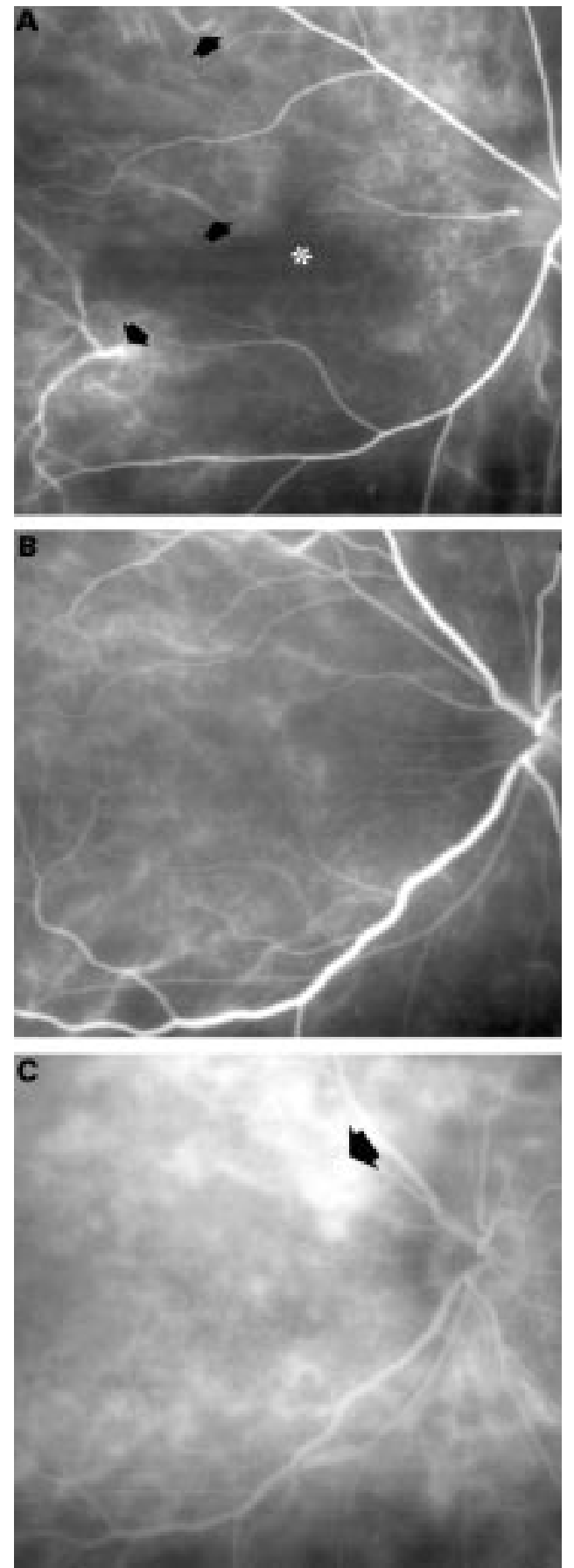

Figure 8 Case 7. Before steroid therapy. Standard ICGA. (A) 17.6 seconds, (B) 31.5 seconds. Choroidal arteries are visualised (arrows), followed by visualisation of

choriocapillaris and choroidal veins. Choroidal filling delay corresponding to watershed zone is clearly observed

(asterisk). (C) 14 minutes 1.8 seconds. The contour of the choroidal vessels is obscured. Intrachoroidal ICG leakage is clearly seen temporal to the optic disc (arrow).

was not observed (Fig 11). Standard ICGA visualised delayed filling of the choroid in the early phase, which faded away after 6 seconds. Some choroidal arteries were dilated and tortuous. The choroidal vessels were irregular, and their contours became vague after 1 minute, with bright fluorescence of varying intensities in the posterior pole (Fig 12). Subtraction of ICG images by the second allowed clear visualisation of delayed filling of 


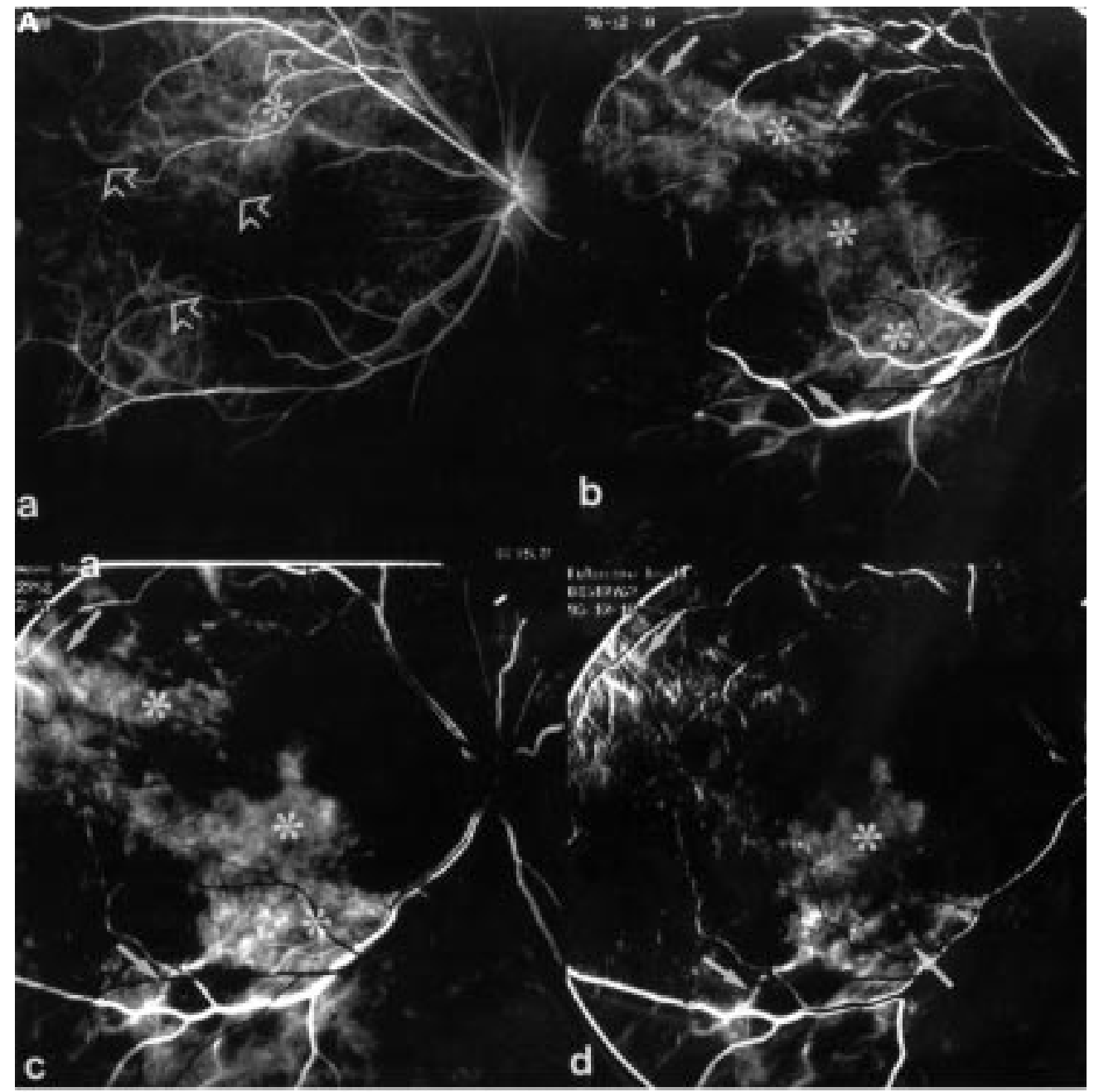

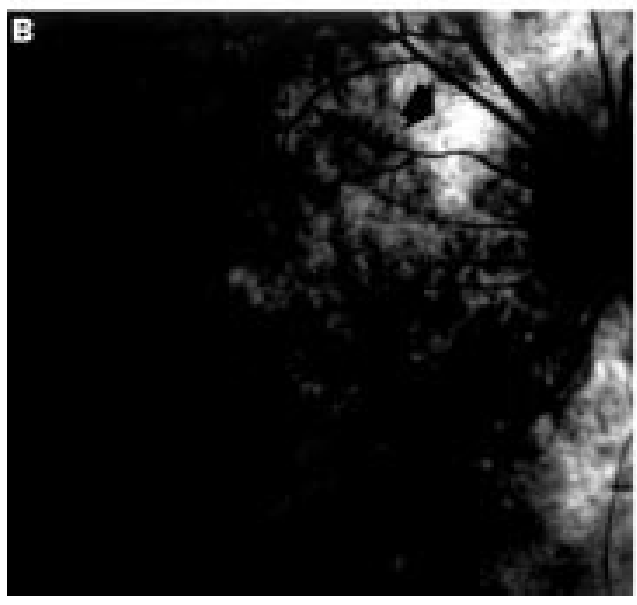

the choriocapillaris and choroidal veins (Fig 13A). Subtraction by the minute revealed bright fluorescence corresponding to ICG leakage (Fig 13B).

\section{Discussion}

In the acute stage of Harada's disease, the presence of delayed choroidal filling has been reported on the basis of findings from FA in the early phase. ${ }^{12}{ }^{13}$ ICGA appears to shed further light on choroidal circulatory disturbances. However, hypoperfusion in the choroidal arteries and choriocapillaris is currently the
Figure 9 Case 7. Before steroid therapy. Subtraction ICGA. (A) Subtraction with an interval of seconds. (a) 19.8-17.6 seconds, (b) 22.6-19.8 seconds, (c) 25.0-22.6 seconds, (d) 28.0-25.0 seconds. (a) Choroidal arteries (open arrows) and choriocapillaris (asterisk) are

visualised. (b-d) Choriocapillaris (asterisks) and choroidal veins (arrows) are delineated in the serial images. The course of the choroidal veins is irregular and discontinuous. (B) Subtraction with an interval of minutes (14 minutes 1.8-42.6 seconds). Bright fluorescence, corresponding to intrachoroidal ICG leakage, is observed (arrow).

only major abnormality that has been clarified by published angiographic studies..$^{3-8}$ Details of the circulatory disturbance in the choroidal veins are still unknown.

In the current study, subtraction of serial angiograms with an interval of about 2 seconds first allowed visualisation of the choroidal arteries, while choriocapillaris and choroidal veins were visualised in subsequent images in the acute stage of disease (Table 2). After steroid therapy, choroidal veins as well as arteries were visualised early in the initial subtraction, and an improvement was noted in the delayed 

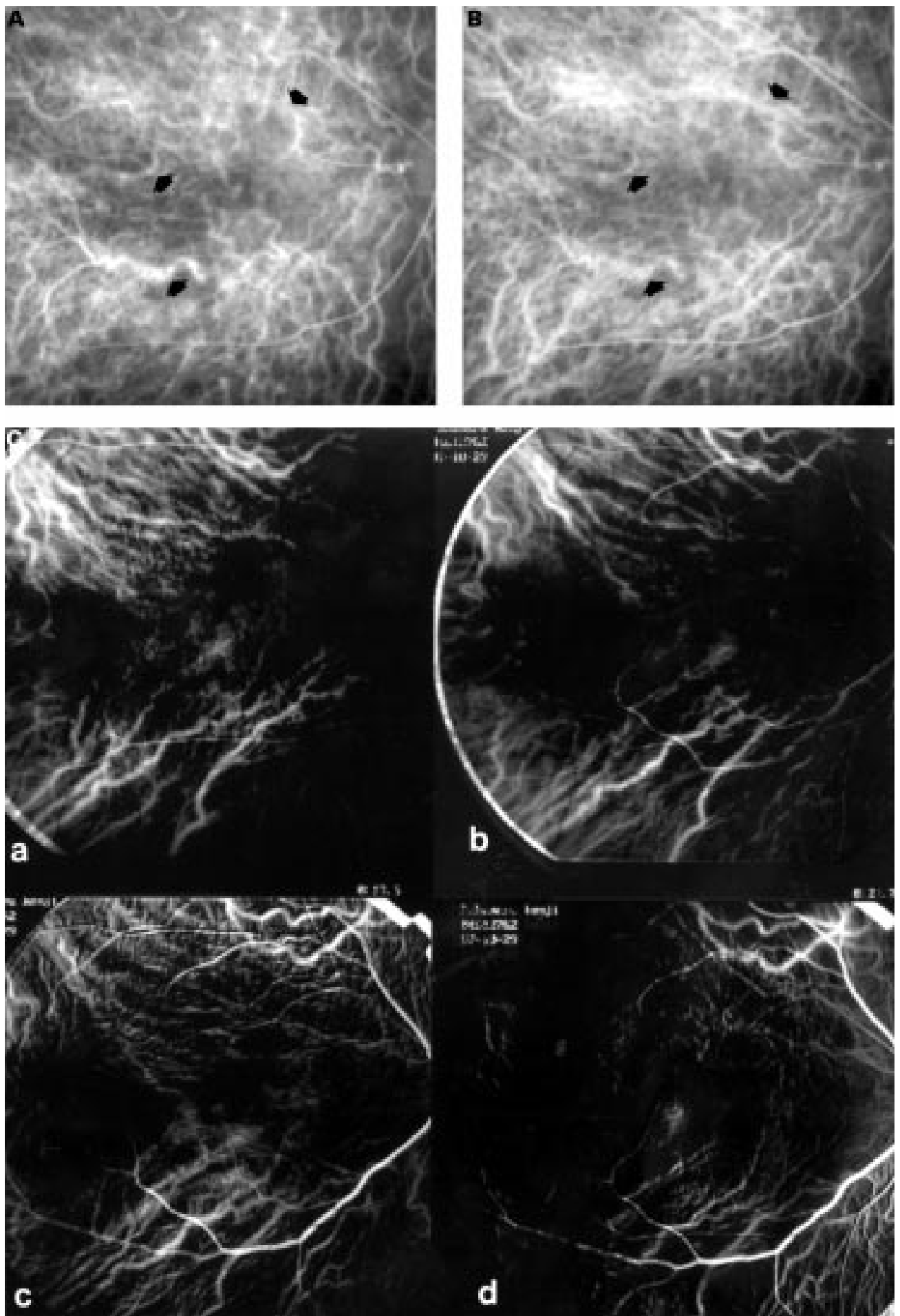

Figure 10 Case 7.10 months on steroid therapy. (A, B) Standard ICGA. (A) 17.8 seconds, (B) 19.6 seconds. Choroidal veins (arrows) as well as arteries are visualised in the initial image. Choroidal filling delay corresponding to the watershed zone has disappeared. The number of visible choroidal vessels has increased. The choroidal veins are more straight, are relatively uniform in calibre, and continuous from the posterior pole to the peripheral region. (C) Subtraction ICGA. (a) 19.6-17.8 seconds, (b) 21.7-19.6 seconds, (c) 24.3-21.7 seconds, (d) 26.3-24.3 seconds. Choroidal veins are visualised in the initial subtracted image. Choroidal venous filling has improved in the serial angiograms.

filling of the choroidal veins. Thus, delayed filling of various degrees was found to be present also in the choroidal veins along with hypoperfusion in the choroid. This improvement in choroidal venous flow appears to be a relatively late phenomenon, as delayed choroidal venous filling was still observed although the serous retinal detachment had disappeared.
As to the cause of the impaired flow into the choroidal veins in Harada's disease, this may be secondary to the marked hypoperfusion of the choroidal arteries. In patients with Takayasu's disease with arterial hypotension, morphological changes, such as narrowing of the choroidal veins as well as delayed choroidal filling during long term follow up, have been reported. ${ }^{14}$ 


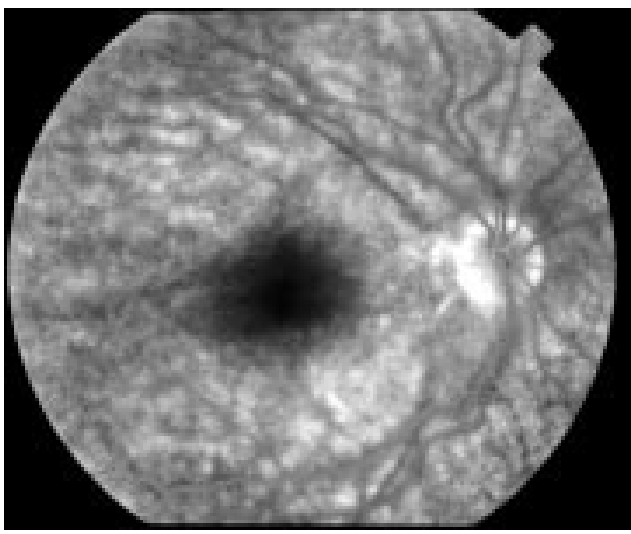

Figure 11 Case 8. FA (2 minutes). No fluorescein dye leakage is seen.

However, in the patients with Harada's disease examined in this study, the choroidal veins appeared irregular in the acute stage. This was associated with delayed venous filling. As the disease subsided, the course of the choroidal veins straightened and became continuous from the posterior pole to the peripheral region, with less variation in calibre. Histopathological studies of Harada's disease have revealed that the choroid is markedly oedematous and infiltrated by inflammatory cells, such as lymphocytes, monocytes, and megakaryocytes. $^{15}{ }^{16}$ These inflammatory changes may be associated not only with circulatory changes with increased permeability of vessels, ${ }^{17}$ but also may generate mechanical compression of the choroidal veins. Thus, a disorder of the choroidal veins as well as choroidal arterial hypoperfusion resulting from decreased inflow pressure is probably responsible for the impaired or delayed choroidal filling in Harada's disease.

ICGA permits the detection of hyperpermeability of the choroidal vessels, resulting in intrachoroidal ICG leakage. ${ }^{19} 19$ However, some reports have focused on hypofluorescence, with a limited description of hyperfluorescent findings. ${ }^{3478}$ Although intrachoroidal ICG leakage has been detected also in Harada's disease, ${ }^{56}$ the accurate evaluation of hyperfluorescence is difficult. Since the excitatory light and the fluorescence transverse a great number of different types of tissues on ICGA ${ }^{20}$ the markedly thickened choroid in the early stage of Harada's disease may affect the brightness of angiograms. Also, the basic design of the Topcon ICGA device itself - that is, the necessity to adjust the emission light intensity according to the brightness of the image obtained, complicates the objective assessment of hyperfluorescence.

ICGA visualises fluorescence emitted from ICG dye present mainly inside blood vessels in the early phase. $^{21}$ Intravascular ICG dye decreases with time, passing through the choriocapillaris into the adjacent interstitial tissue of the choroid. Consequently, the choroidal vessels are visualised in the early phase; then ICG leakage is observed in the mid phase. Subtraction of angiograms with an interval of minutes between the early phase and the mid phase reveals abnormal ICG leak-
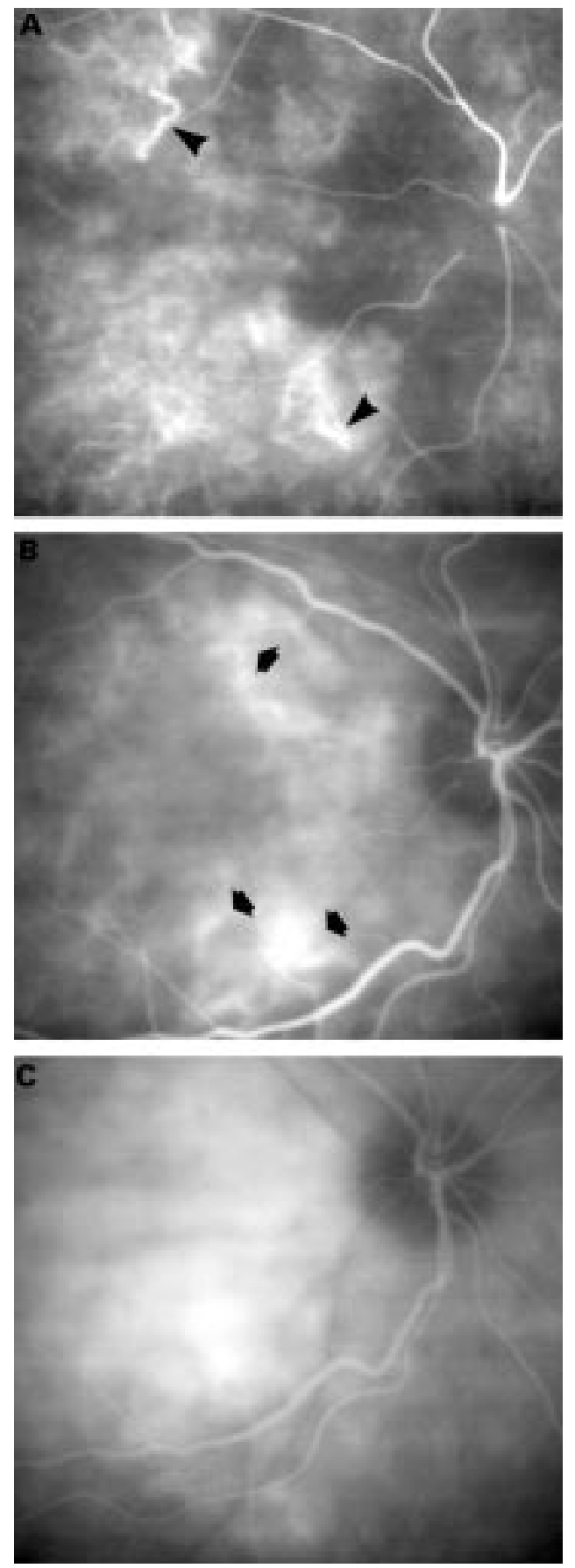

Figure 12 Case 8. Standard ICGA. (A) 24.8 seconds, (B) 39.5 seconds. Delayed choroidal filling is seen at the posterior pole. Choroidal arteries are dilated and tortuous (arrowheads). The course of the choroidal vessels is irregular and the contour becomes obscure with time (arrows). (C) At 12 minutes 0.2 seconds, hyperfluorescence of various intensity is seen over the whole area of the posterior pole.

age specifically as positive images separated from normal areas. ${ }^{11}{ }^{11}$ In the present study, hyperfluorescent areas corresponding to intrachoroidal ICG leakage were found on the standard angiograms in all cases examined; on subtraction by the minute, portions of the hyperfluorescent areas were visualised as positive images. As the disease subsided with steroid therapy, these positive findings disappeared. Thus, we believe that the presence of 

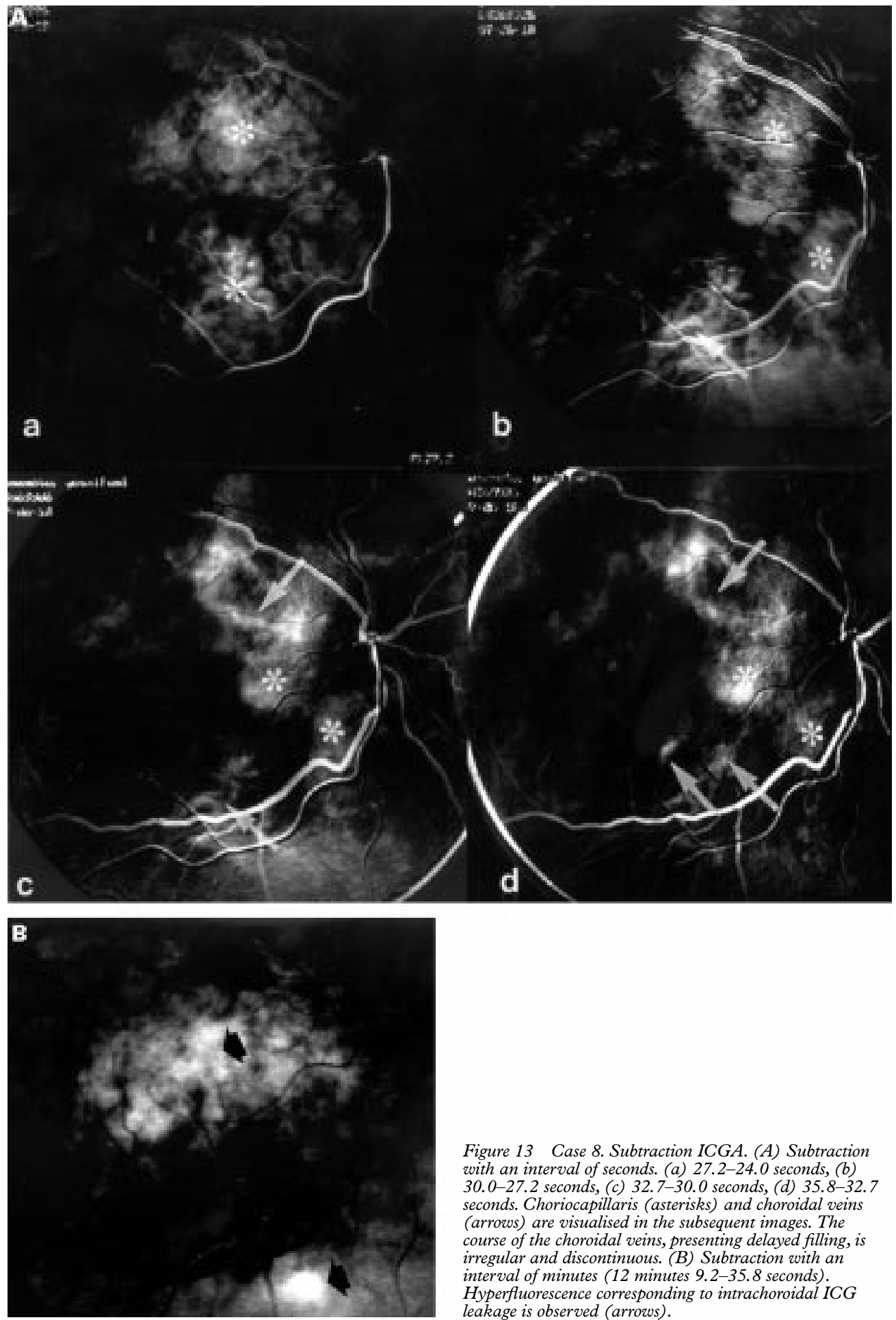

Figure 13 Case 8. Subtraction ICGA. (A) Subtraction with an interval of seconds. (a) 27.2-24.0 seconds, (b) 30.0-27.2 seconds, (c) 32.7-30.0 seconds, (d) 35.8-32.7 seconds. Choriocapillaris (asterisks) and choroidal veins (arrows) are visualised in the subsequent images. The course of the choroidal veins, presenting delayed filling, is irregular and discontinuous. (B) Subtraction with an interval of minutes (12 minutes 9.2-35.8 seconds). Hyperfluorescence corresponding to intrachoroidal ICG leakage is observed (arrows).

Table 2 Choroidal abnormalities in acute stage of the disease

\begin{tabular}{|c|c|c|c|c|c|c|c|c|c|}
\hline \multirow[b]{2}{*}{$\begin{array}{l}\text { Case } \\
\text { No }\end{array}$} & \multicolumn{2}{|l|}{$F A$} & \multicolumn{4}{|c|}{ Standard ICGA } & \multicolumn{3}{|l|}{ Subtraction ICGA } \\
\hline & $\begin{array}{l}\text { Choroidal } \\
\text { filling delay }\end{array}$ & $\begin{array}{l}\text { Fluorescein } \\
\text { leakage }\end{array}$ & $\begin{array}{l}\text { Choroidal } \\
\text { filling delay }\end{array}$ & $\begin{array}{l}\text { Tortuous and } \\
\text { dilated choroidal } \\
\text { arteries }\end{array}$ & $\begin{array}{l}\text { Vascular } \\
\text { irregularity }\end{array}$ & $\begin{array}{l}\text { Intrachoroidal } \\
\text { ICG leakage }\end{array}$ & $\begin{array}{l}\text { Sequential image } \\
\text { of choroidal } \\
\text { vessels }\end{array}$ & $\begin{array}{l}\text { Slow filling of the } \\
\text { choroidal veins }\end{array}$ & $\begin{array}{l}\text { Smaller area of } \\
\text { intrachoroidal } \\
\text { ICG leakage }\end{array}$ \\
\hline 1 & + & - & + & + & + & + & + & + & + \\
\hline 2 & + & + & + & + & + & + & + & + & + \\
\hline 3 & + & + & + & + & + & + & + & + & + \\
\hline 4 & + & + & + & - & + & + & + & + & + \\
\hline 5 & + & + & + & + & + & + & + & + & + \\
\hline 6 & + & + & + & - & + & + & + & + & + \\
\hline 7 & + & + & + & - & + & + & + & + & + \\
\hline 8 & + & - & + & + & + & + & + & + & + \\
\hline
\end{tabular}


hyperpermeability of the choroidal vessels has been confirmed in Harada's disease by the subtraction method. In subtraction studies of choroidal neovascularisation associated with age related macular degeneration, two types of ICG leakage have been reported-one visualised by the subtraction method and the other not visible on subtraction-indicating a relation with the degree of hyperpermeability of the site of choroidal neovascularisation. ${ }^{11}$ In Harada's disease, areas of the choroidal vessels with intense hyperpermeability appear to be visualised as positive images.

In conclusion, subtraction ICGA has highlighted inflow changes of the choroidal veins and demonstrated intrachoroidal ICG leakage in Harada's disease. These results indicate that delayed filling of the choroidal veins of varying degrees of severity occurs in association with hyperpermeability of the choroidal vessels in the course of the disease.

This study was supported in part by the Osaka Eye Bank Association Fund, Osaka, Japan.

This paper was presented at the International Congress of Ocular Circulation and Neovascularisation in Kyoto, 1997.

1 Flower RW, Hochheimer BF. A clinical technique and apparatus for simultaneous angiography of the separate retinal and choroidal

2 Bischoff PM, Flower RW. Ten years' experience with choroidal angiography using indocyanine green dye: a new routine examination or an epilogue? Doc Ophthalmol 1985 60:235-91.

3 Yuzawa $M$, Kawamura A, Matsui M. Indocyanine green video-angiographic findings in Harada disease. $\mathrm{Fpn}^{\mathrm{F} O \mathrm{Oph}}$ thalmol 1993;37:456-66.

4 Matsunaga H, Matsubara T, Fukushima I, et al. Indocyanine green fluorescence angiography in Harada disease. $f$ Fpn Ophthalmol Soc 1994;98:852-7.

5 Kohno T, Miki T, Shiraki K, et al. ICG angiographic evaluation of intrachoroidal vascular abnormalities associated with Harada's disease. Proceedings of the Second Inter- national Symposium on Indocyanine Green Angiography 1995:57-9.

6 Iida T, Hagimura N, Otani T, et al. Choroidal lesions in serous retinal detachments by indocyanine green angiography. Proceedings of the Second International Symposium on Indocyanine Green Angiography 1995:63-5.

7 Ohshima Y, Harino S, Hara Y, et al. Indocyanine green angiographic findings in Vogt-Koyanagi-Harada disease. Am 7 Ophthalmol 1996;122:58-66.

8 Pece A, Bolognesi G, Introini U, et al. Indocyanine green angiography in Vogt-Koyanagi-Harada-type disease. Arch Ophthalmol 1997;115:804-6.

9 Flower RW. Extraction of choriocapillaris hemodynamic data from ICG fluorescence angiograms. Invest Ophthalmol Vis Sci 1993;34:2720-9.

10 Miki T, Shiraki K, Kohno T, et al. Computer assisted image analysis using the subtraction method in indocyanine green angiography. Eur f Ophthalmol 1996;6:30-8.

11 Kohno T, De Laey JJ, Miki T. Detection of choroidal neovascularization in age-related macular degeneration using subtraction methods in indocyanine green angiography. Bull Soc Belge Ophtalmol 1995;259:81-8.

12 Shimizu K. Fluorescein microangiography of the ocular fundus. Tokyo: Igaku-Shoin, 1974:91-100.

13 Young NJA, Bird AC, Sehmi K. Pigment epithelial diseases with abnormal choroidal perfusion. Am $\mathcal{f}$ Ophthalmol 1980;90:607-18.

14 Sutoh N, Muraoka K, Takahashi K, et al. Indocyanine green angiography in Takayasu disease. fpn $\mathcal{f}$ Clin Ophthalmol 1995;49:1811-19.

15 Ikui H, Hiroishi M, Furuyoshi Y. Histopathology of idiopathic uveitis. Report of two cases. Acta Soc Ophthalmol 7pn 1952;56:1079-91.

16 Green WR. Vogt-Koyanagi-Harada (VKH) syndrome. In: Spencer WH, ed. Ophthalmic pathology. An atlas of text book. Philadelphia. Saunders, 1986:1956-66.

17 Cotran RS, Kumar V, Robbins SL. Inflammation and repair. Inflammation. In: Cotran RS, Kumar V, Robbins SL, eds. Robbins pathologic basis of disease. Philadelphia: Saunders, 1989:39-71.

18 Kohno T, Miki T, Kitashoji K. Indocyanine green videoangiography of the choroidal circulation in the experimental retinal opacity induced by blunt trauma. Acta Soc Ophthal mol fpn 1992;96:749-56.

19 Miki T, Kitashoji K, Kohno T. Intrachoroidal dye leakage in Miki T, Kitashoji K, Kohno T. Intrachoroidal dye leakage in
indocyanine green fundus angiography after experimental commotio retinae. Eur $\mathcal{f}$ Ophthalmol 1992;2:79-82.

20 Flower RW. Physical optics concepts related to obtaining and interpreting ICG angiograms. In: Yannuzzi LA, Flower RW, Slakter JS, eds. Indocyanine green angiography. St Louis: Mosby, 1997:105-18.

21 Flower RW. Choroidal fluorescent dye filling patterns. A comparison of high speed indocyanine green and fluorescein angiograms. Int Ophthalmol 1980;2,3:143-9. 